\title{
Tomoscintigraphy for detecting gastrointestinal and medullary thyroid cancers: first clinical results using radiolabelled monoclonal antibodies against carcinoembryonic antigen
}

\author{
CHRISTIAN BERCHE，JEAN-PIERRE MACH，JEAN-DENIS LUMBROSO, CATHERINE LANGLAIS, \\ FLORENT AUBRY, FRANZ BUCHEGGER, STEPHAN CARREL, PHILIPPE ROUGIER, \\ CLAUDE PARMENTIER, MAURICE TUBIANA
}

\begin{abstract}
Transaxial tomoscintigraphy (or single-photon emission computerised tomography) was used to detect secondary deposits of carcinoma in 17 patients who had been injected with iodine-131-labelled monoclonal antibodies against carcinoembryonic antigen. Of 17 tumour sites studied by tomoscintigraphy 16 were detected (sensitivity 94\%); five sites had a volume smaller than $10 \mathrm{~cm}^{3}$. Tomoscintigraphy also detected three unknown tumour deposits later confirmed by surgery or radiology. In contrast, when 21 tumour sites in the same patients were studied by rectilinear scintigraphy, only nine tumour sites were detected (sensitivity $43 \%$ ), of which eight had a volume larger than $50 \mathrm{~cm}^{3}$.
\end{abstract}

Institut Gustave-Roussy and Institut de Recherches de Radiobiologie Clinique, F-94800 Villejuif, France

CHRISTIAN BERCHE, $M D$, assistant physician

JEAN-DENIS LUMBROSO, MD, assistant physician

CATHERINE LANGLAIS, research assistant

FLORENT AUBRY, PHD, research fellow

PHILIPPE ROUGIER, MD, assistant physician

CLAUDE PARMENTIER, MD, professor and head of nuclear medicine department

MAURICE TUBIANA, MD, PHD, professor, chief of radiation department and director of the Institut de Recherches de Radiobiologie Clinique

Ludwig Institute for Cancer Research, 1066 Epalinges, Lausanne, Switzerland

JEAN-PIERRE MACH, MD, associate professor

FRANZ BUCHEGGER, PHD, biochemist

STEPHAN CARREL, MD, assistant physician
As compared with conventional scintigraphy, tomoscintigraphy represents a fundamental improvement which can increase both the sensitivity and specificity of immunoscintigraphy.

\section{Introduction}

Immunoscintigraphy using radiolabelled antibodies against carcinoembryonic antigen (CEA) was performed on animals in $1974^{1-3}$ and on man soon after. ${ }^{4-6}$ Until recently polyclonal anti-CEA antibodies were used in clinical studies. ${ }^{4-8}$ Immunological specificity can be improved with monoclonal anti-CEA antibodies. ${ }^{910}$

When immunoscintigraphy is performed with conventional scintigraphy the tumour contrast can be enhanced by subtracting scintigrams obtained with non-specific radiotracers ${ }^{11}$ from the radioactivity due to the antibody. This subtraction technique presents limitations due, firstly, to differences in the distribution of the antibody and of the non-specific tracers in normal tissues and, secondly, to the difference in energy of the most commonly used radionuclides ( ${ }^{131} \mathrm{I}$ and ${ }^{9{ }^{9 m} \mathrm{~T}} \mathrm{~T}$ ).

Axial transverse tomoscintigraphy ${ }^{12}$ allows reconstruction of the three-dimensional distribution of the radiolabelled tracers. The contrast is related to the ratio between the activity per unit mass of the tumour and that of the normal tissues. For the study of relatively small tumours immunotomoscintigraphy should therefore represent a fundamental improvement over conventional immunoscintigraphy, in which the contrast is volume dependent.

We compared the results obtained with immunotomoscintigraphy and immunoscintigraphy in 19 patients injected with anti-CEA monoclonal antibodies labelled with ${ }^{131} \mathrm{I}$. 


\section{Patients and methods}

Monoclonal anti-CEA antibodies were produced in ascitic form by two mouse hybridoma lines. ${ }^{9} \mathrm{~W}$ ith their informed consent, we included in the study patients without personal or family histories of allergy and with negative responses to tests of hypersensitivity to normal mouse IgG. They were injected intravenously (under cover with anti-allergic drugs) with $0.3 \mathrm{mg}$ of ${ }^{131} \mathrm{I}$-labelled monoclonal antibodies representing $37-55 \mathrm{MBq}(1-1.5 \mathrm{mCi})$. The thyroid was blocked with Lugol's iodine. None of the 19 patients injected with mouse anti-CEA antibodies showed any adverse effect or any sign of discomfort during or after the injection.

The radioactivity of the normal thyroid was measured on surgical specimens of one patient (case 11) two days after the injection of $55.5 \mathrm{MBq}(1.5 \mathrm{mCi})$ of anti-CEA antibodies and was equal to 20 $\mathrm{kBq} / \mathrm{g}(0.54 \mu \mathrm{Ci} / \mathrm{g})$ after correction of physical decay. This was approximately equal to activities found after conventional thyroid scintigraphy with $1 \mathrm{MBq}(27 \mu \mathrm{Ci})$ of ${ }^{131} \mathrm{I}$.

Rectilinear scintigrams were produced with a scintigraph OhioNuclear $84-F D$. Over 40 minutes the anterior view and the sum of
Fifty-one examinations using-tomoscintigraphy in all 19 patients were performed: 29 were immunotomoscintigrams using radiolabelled monoclonal anti-CEA antibodies (17 performed the first day, nine the second day, and three the third day after the injection) and 22 were tomoscintigrams with other radiolabelled compounds (nine with $99 \mathrm{~m} \mathrm{Tc}$-methylen diphosphonate, six with ${ }^{99 \mathrm{~m}} \mathrm{Tc}$-sulfur-colloid, four with ${ }^{99 \mathrm{~m} T c} \mathrm{~T}$-pertechnetate, and three with $\left.{ }^{123} \mathrm{I}\right)$. These other radiolabelled compounds were used to obtain radioactive anatomical landmarks in the same position as with immunotomoscintigraphy. Only 17 of the 22 tumour sites were studied by immunotomoscintigraphy.

\section{Results}

\section{RECTILINEAR SCINTIGRAPHY}

The rectilinear scintigrams allowed us to determine the temporospatial distribution of the injected radiolabelled heterologous antibodies in the normal organs. The vascular radioactivity decreased progressively, with a half life of about 1.5 days: only the heart was

Scintigraphic and tomoscintigraphic results in 19 patients (17 with tumours and two without). Patients are classified according to their primary carcinomas

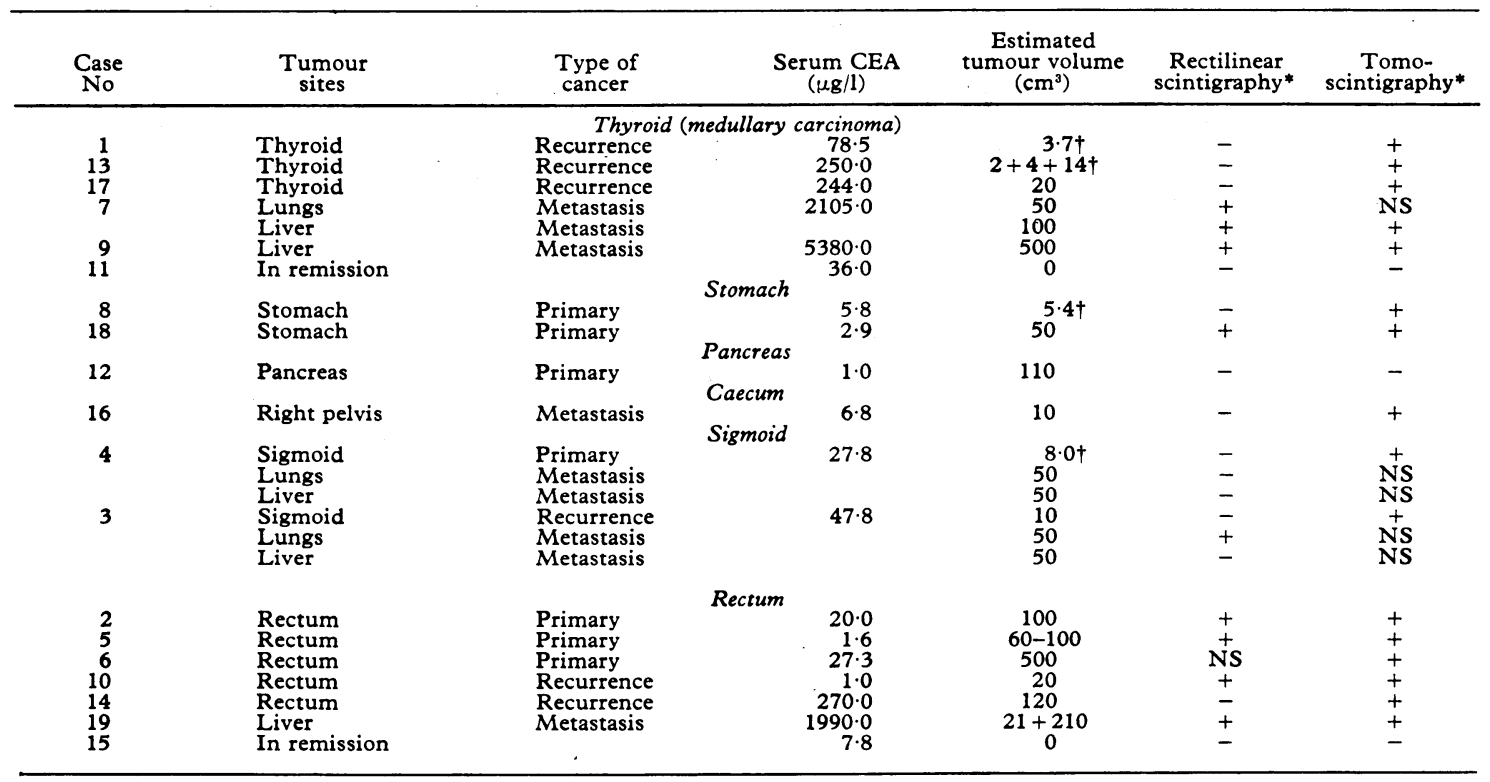

* + = Detected site; - = site not detected; NS = site not studied. + Exact value found after surgery.

anterior and posterior views of the total body (except the legs) were obtained. Owing to the efficiency of this detector for ${ }^{131} \mathrm{I}$, it was possible to perform such examinations up to eighteen days after the antibody injection.

For tomoscintigraphy we used our tomographic prototype. It comprises a scintillation camera with a large field of view (Acticamera CGR) which rotates around the patient as he lies on a specially designed bed. The rotation time can vary from 1 to 20 minutes for an entire turn. The collected data are stored on a minicomputer Simis 5 Informatek and are processed with original algorithms ${ }^{13} 14$ which enable the reconstruction of transverse sections or any other plane sections (frontal, sagittal, or oblique). Immunotomoscintigraphy could be performed up to three days after the injection of monoclonal antibodies.

Nineteen patients with a history of primary cancer were studied: six had medullary thyroid carcinomas and 13 gastrointestinal carcinomas (see table). Two patients (cases 11 and 15) who had had their primary carcinomas removed ( 1 thyroid and 1 rectum carcinoma) were in complete clinical remission at the time of examination. The other 17 patients presented 22 major tumour sites detected by other techniques: surgery, computed tomography, echography, radiography, and conventional scintigraphy.

Forty-five examinations using rectilinear scintigraphy (in the two patients without tumours and in 16 of those with tumours) were carried out five hours to 18 days after injection. One patient with one tumour site was too tired to undergo this examination. slightly visible at day 8 , and the kidneys and bladder disappeared at day 4. Despite the treatment with iodine, the thyroid was visible on day 1 and thyroid uptake at day 2 was maximal and higher than any other uptake. The activity in the reticuloendothelial system (liver, spleen, bone marrow) was greatest on day 1 ; thereafter it decreased and disappeared at day 4.

The sensitivity (percentage of tumour sites detected) of rectilinear scintigraphy for detecting tumours was assessed by double-blind readings performed by four physicians. The average of their results showed that rectilinear scintigraphy detected nine of the 21 tumour sites studied in 16 patients ( $43 \%$ sensitivity). Out of 13 tumour sites with a volume larger than $50 \mathrm{~cm}^{3}$, eight were detected ( 3 in livers, 2 in lungs, 1 in the stomach, 2 in rectums; table). Of these eight, four ( 2 in rectums, 1 in the lung, 1 in the stomach) seemed clearer on the later scintigrams (on days 4 to 8 ).

Four areas of abnormal non-specific uptake were noted in four patients with tumours: three of the areas were of unknown origin (1 in the liver, 1 in the stomach, 1 in the right pelvis) and one secondary to an operation on the stomach (case 12).

\section{TOMOSCINTIGRAPHY}

Fig 1 shows the results obtained in two patients with local recurrences of thyroid medullary epitheliomas injected with ${ }^{131}$ I-anti-CEA antibodies. One patient (case 1) was suspected of having a recurrence 
15 years after a left hemithyroidectomy and postoperative irradiation because of an increase in serum CEA concentration to $78.5 \mu \mathrm{g} / \mathrm{l}$ (normal $<5 \mu \mathrm{g} / \mathrm{l}$ ). Fig $1 a$ and Fig $1 b$ show, respectively, transverse and frontal sections on day 1 passing through a tumour recurrence in the right lobe, which was shown to weigh $3.7 \mathrm{~g}$ at subsequent surgery. Histological examination showed that the thyroid was almost exclusively cancerous, with rare foci of sclerotic thyroid tissue. Thus, the right thyroid uptake observed on the sections was due to tumour and not to uptake of free ${ }^{131} I$ in normal thyroid.

In case 17 carcinoma of the right thyroid lobe was treated by local irradiation (70 Grays (7000 rads)) one year before examination. Recurrence was suspected because of a recent rise in serum CEA concentration to $244 \mu \mathrm{g} / \mathrm{l}$. Fig $1 c$ and Fig $1 d$ show transverse sections at the same level on the middle part of the thyroid and were obtained with monoclonal anti-CEA antibodies $(c)$ and ${ }^{123} \mathrm{I}(d)$ on day 2 . The radioactive distributions were clearly different: the maximum uptake of ${ }^{131} \mathrm{I}$ corresponded to the tumour recurrence $(c)$ and was located on
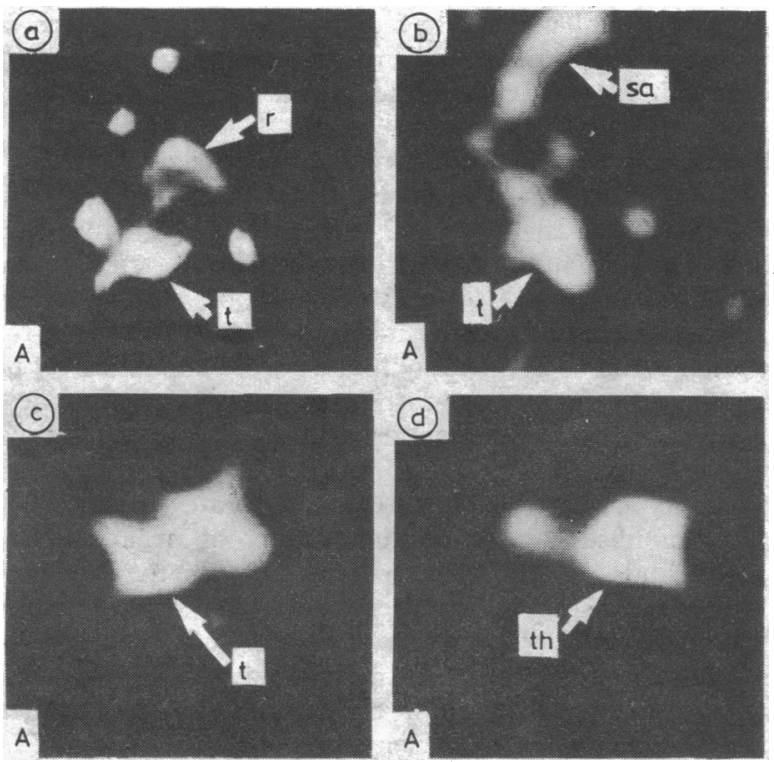

FIG 1-Tomoscintigrams performed with ${ }^{131}$ I-anti-CEA mononuclear antibody in two patients with local recurrence of medullary thyroid carcinoma (A denotes front and right). Case 1. $a$ and $b$ show, respectively, transverse and frontal sections (zoom 1.8) at day 1 of patient with $3 \cdot 7-\mathrm{g}$ tumour of right lobe of thyroid, 15 years after left hemithyroidectomy of primary tumour and local irradiation. Case 17. $c$ and $d$ show transverse sections at same level on middle part of thyroid (zoom 2) on day 2 in patient with tumour recurrence in right lobe of thyroid. $c$ was obtained with ${ }^{131} \mathrm{I}$-anti-CEA antibodies and $d$ with ${ }^{123} \mathrm{I}$. Tumour is seen on the right lobe of thyroid, where ${ }^{123} I$ uptake is almost absent. $t=$ tumour; $r=$ cervical rachis; $s a=$ salivary glands; th=normal residual thyroid tissue.
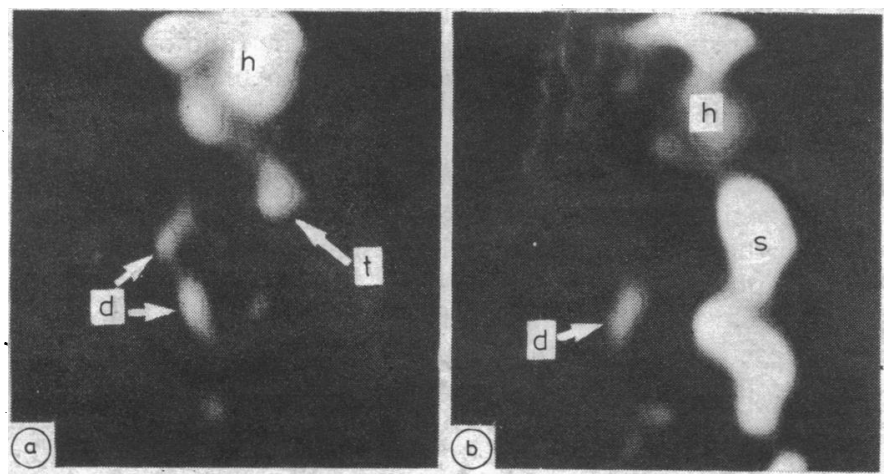

FIG 2-Case 8. Tomoscintigrams of primary carcinoma of stomach (frontal sections at same level through stomach). $a$ was obtained with ${ }^{131}$ I-anti-CEA monoclonal antibody on day 3 and $b$ with ${ }^{99} \mathrm{~m} \mathrm{Tc}$-pertechnetate. The 5.4-g tumour $(t)$ seen in $a$ could be localised on the lesser curvature of the stomach (s) in $b . h=$ heart; $d=$ duodenum with benign ulcer, visible owing to bleeding.
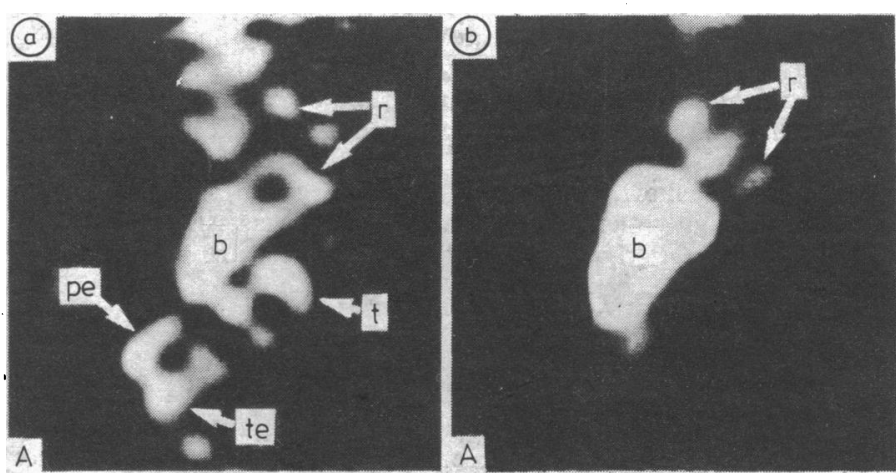

FIG 3-Case 5. Tomoscintigrams of primary carcinoma of rectum (sagittal sections at same level, $A$ denotes the front). $a$ was obtained with ${ }^{181}$ I-antiCEA antibodies on day 1 . The $6-\mathrm{cm}$ tumour $(t)$ was seen behind the bladder (b). $b$ shows a bone tomoscintigram with ${ }^{99 \mathrm{~m}} \mathrm{Tc}$-methylen diphosphonate in the same position as shown by immunotomoscintigram. This section showed no ${ }^{99 m} \mathrm{Tc}$ uptake in the tumour area behind the bladder. Rachis (r) was detected on both scintigrams, and non-specific uptakes on the penis (pe) and testes (te) are seen in $a$.
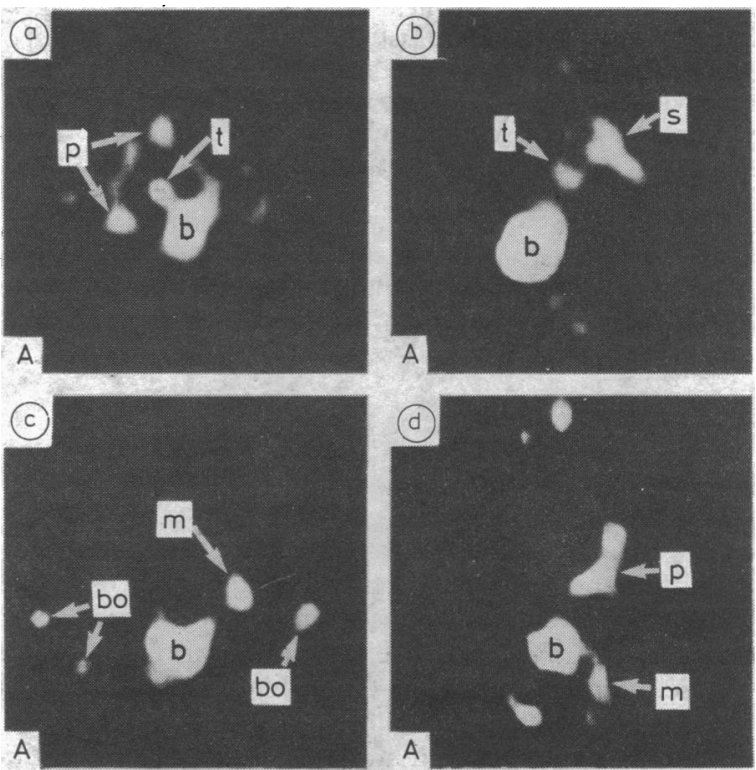

FIG 4-Case 4. Tomoscintigram with 131I-anti-CEA antibodies on day 1 of primary carcinoma of sigmoid (A denotes front and right). $a$ and $b$ show, respectively, transverse and sagittal sections passing through 8-g primary tumour $(\mathrm{t})$ located between bladder (b) and sacrum (s) or pelvic bones (p). $c$ shows transverse section at lower part of bladder passing through lymph-node metastasis (m) on left side of rectum. It also shows non-specific bone marrow uptakes (bo). $d$ shows sagittal section passing through same lymph node metastasis, which is-located behind bladder and below pelvic bones.

the right lobe of the thyroid, whereas there was almost no ${ }^{123}$ I uptake in this area $(d)$. The remaining normal left lobe of the thyroid showed a definite uptake of ${ }^{123} \mathrm{I}$.

Fig 2 shows frontal sections at the same level passing through the middle of the stomach in a patient with a primary carcinoma of the lesser curvature of the stomach (case 8). Fig $2 a$ was obtained with ${ }^{131}$ I-anti-CEA antibodies on day 3 and fig $2 b$ with ${ }^{99}$ Tc-pertechnetate to visualise the stomach. Tumour was seen on $a$ and could be localised to the lesser curvature of the stomach delineated by ${ }^{99 \mathrm{~m}} \mathrm{Tc}(b)$. At subsequent operation the tumour weighed $5.4 \mathrm{~g}$.

The patient shown in fig 3 (case 5) had a primary rectal carcinoma of 6-cm spread. Fig 3 shows sagittal sections at the same level obtained with ${ }^{181} \mathrm{I}$-anti-CEA antibodies and ${ }^{\circ 9 \mathrm{~m}} \mathrm{Tc}$-methylen diphosphonate. The tumour was seen on $a$ behind the bladder. The tumour uptake did not correspond to any ${ }^{\circ 9 \mathrm{~m}} \mathrm{Tc}$ uptake as shown in $b$. On the rachis the antibody and methylen diphosphonate bone uptakes did not have exactly the same distribution because the antibodies were fixed on bone marrow. 
Fig 4 shows the results of immunotomoscintigraphy on day 1 in case 4-a patient with an untreated primary sigmoid carcinoma. Fig $4 a$ and fig $4 b$ show, respectively, transverse and sagittal sections passing through the primary tumour located between the bladder and the sacrum or the pelvic bones. At subsequent surgery the primary tumour was resected and found to weigh $8 \mathrm{~g}(2.5-\mathrm{cm}$ diameter $)$. Fig $4 c$ and fig $4 d$ show, respectively, transverse and sagittal sections passing through a left rectal lymph-node metastasis detected by immunotomoscintigraphy and confirmed by subsequent surgery.

\section{SENSITIVITY}

For evaluating the sensitivity of tomoscintigraphy double-blind readings were performed by three observers. Of the 17 tumour sites studied, 16 were detected (see table). The only undetected site was a primary carcinoma of the pancreas with a volume of about $110 \mathrm{~cm}^{3}$. In this patient (case 12) the serum CEA concentration was $1 \mu \mathrm{g} / 1$.

Of the 16 tumour sites in 16 patients studied by both tomoscintigraphy and rectilinear scintigraphy, 15 sites were detected by tomoscintigraphy ( $94 \%$ sensitivity) compared with seven detected by rectilinear scintigraphy ( $44 \%$ sensitivity).

Moreover, for three patients with tumours immunotomoscintigraphy revealed areas of uptake which did not correspond to a previously known tumour site. At subsequent surgery two of these uptakes were found to be lymph-node metastases (see fig 4). The third corresponded to a bone metastasis which was confirmed by $x$-ray examination. These previously unknown tumour sites were not taken into account for assessing the diagnostic efficiency.

The observers noted seven areas of abnormal radioactive uptake (six in five patients with tumours and one in one without) which did not correspond to known tumour deposits. Three of these areas were related to a major gastrointestinal operation (out of eight such operations) and one other to a bleeding duodenal ulcer (fig 2). The remaining three areas (stomach, left sacrum, liver) were not explained by any known lesion.

\section{Discussion}

\section{SENSITIVITY OR TRUE-POSITIVE RATE}

Ninety-four per cent of the tumour sites studied by the two methods were detected by tomoscintigraphy compared with $44 \%$ by rectilinear scintigraphy. The percentage of tumours detected by tomoscintigraphy was also much higher than the $50 \%$ (14 out of 28 gastrointestinal tumours) obtained by Mach et al, ${ }^{10}$ who used the same labelled monoclonal anti-CEA antibodies with conventional scintigrams and a subtraction technique."11 Moreover, the four observers of the rectilinear scintigrams presented divergent results because of the poor tumour contrast in the scintigrams, and we had to consider a rectilinear scintigram as showing a positive finding when at least two observers considered it so. This probably enhanced the results for rectilinear scintigraphy compared with those for tomoscintigraphy, for which the responses of the three observers were concordant.

Furthermore, rectilinear scintigraphy mainly detected tumours larger than $50 \mathrm{~cm}^{3}$ whereas tomoscintigraphy showed tumours as small as a few $\mathrm{cm}^{3}$. Detection by tomoscintigraphy seems to be relatively independent of tumour size (except perhaps for very small tumours) but dependent on the concentration of labelled monoclonal antibodies in the tumour as compared with adjacent normal tissues. The only tumour site not visualised by tomoscintigraphy (case 12) was large $\left(110 \mathrm{~cm}^{3}\right)$, and this negative result was probably due to a low concentration of antibody in the tumour. This result and the low serum CEA concentration of this patient suggested that this tumour was synthetising very little CEA.

\section{SPECIFICITY AND FALSE-POSITIVE RESULTS}

In four patients with tumours a surgical procedure was carried out after antibody injection, and the radioactivity of resected tumour tissues was found to be 1.4 to 22 times higher than that of normal adjacent tissues and 1 to 5 times lower than that of sera. These findings confirmed a specific uptake of anti-CEA monoclonal antibodies in the tumour. ${ }^{10}$

Non-specific uptakes of anti-CEA antibodies do exist, however, and remain a problem. When located near the site of a tumour these non-specific uptakes can be interpreted as truepositive results-for example, the most evident false-positive finding on rectilinear scintigraphy corresponded to non-specific uptake in the stomach (probably due to a previous operation) front of the pancreatic tumour (case 12). It was detected by three physicians (out of four) and was scored as positive. In fact tomoscintigraphy showed that the uptake was not in the tumour but in the stomach. In addition, at least three other tumour sites (three rectums) detected by rectilinear scintigraphy corresponded to superimpositions of tumour uptakes with non-specific uptakes (see fig 3). Several so-called tumour uptakes on conventional scintigraphy might be due to non-specific uptakes. Thus we think that the actual sensitivity of conventional immunoscintigraphy might be lower than that reported ${ }^{4}{ }^{7}{ }^{7}$ : some so-called true-positive results might in fact be false-positive. Such artefacts might also partially explain the surprisingly low number of false-positive results reported by some authors ${ }^{7}$ with standard scintigraphy.

The number of abnormal non-specific uptakes (seven in five patients with tumours and one without) detected by tomoscintigraphy was higher than for rectilinear scintigraphy (four in four patients with tumours). Nevertheless, only one (left sacrum) of the seven false-positive uptakes detected by tomoscintigraphy was higher than the true-positive uptakes detected by tomoscintigraphy and four of these uptakes were explained by using landmark tomoscintigraphy with conventional radionuclides (fig 2). Hence, with complementary tomoscintigraphy, we could exclude four false-positive results.

Specificity is related to the false-positive rate (specificity $=1-$ (number of false-positive results/number of negative zones)), which depends on the number of negative patients or zones? studied. Obviously, the number of patients without tumours in this study (two) was too low for a conventional evaluation. The patients with tumours may, however, be considered negative when we exclude their tumour areas. With this method the specificity of rectilinear scintigraphy was $78 \%(1-(4 / 18))$ and the specificity of tomoscintigraphy varied from $63 \%(1-$ $(7 / 19))$ to $84 \%(1-(3 / 19))$ according to whether or not we took account of the results of the complementary landmark tomoscintigraphy.

For an overall diagnostic evaluation we used an index called gain, ${ }^{14}$ which is the percentage of the true-positive results (sensitivity) minus the percentage of the false-positive results. The gain can be interpreted as the value of sensitivity when there is no false-positive result. Thus, the gain with rectilinear scintigraphy was weak (22\%) compared with the gain with tomoscintigraphy, which varied from $57 \%$ to $78 \%$ according to whether complementary tomoscintigraphy was taken into account.

The gain with tomoscintigraphy is essentially dependent on the false-positive rate, which is related to the ratio of uptake in the tumour to non-specific uptake. Fragments of polyclonal antibodies or of monoclonal antibodies (such as $F\left(a^{\prime}\right)_{2}$ fragments ${ }^{10}$ ) might be better tracers than the antibodies in current use. Other radionuclides with shorter physical half lives than ${ }^{131}$ I and emitting photons with lower energy, such as ${ }^{123} \mathrm{I}$ or ${ }^{111} \mathrm{In}$, may further increase the spatial resolution of immunotomoscintigraphy.

In conclusion, the clinical use of rectilinear scintigraphy for immunoscintigraphy with anti-CEA antibodies seems limited to the detection of large tumours, which are often best appreciated on late scintigrams (days 4 to 8 ). Immunotomoscintigraphy, in contrast, allows the detection of smaller tumours, and it can be used even where non-specific uptakes are important, especially on days 1 and 2. Thus, immunotomoscintigraphy with anti-CEA monoclonal antibodies may represent an efficient diagnostic technique for detecting occult CEA-producing tumours pre- 
operatively, and, in particular, for localising recurrences or metastases of medullary thyroid carcinomas.

This work was supported in part by clinical research grant 80-A4 from the Institut Gustave-Roussy. Requests for reprints should be addressed to Dr C Berche, Service de Médecine Nucléaire, Institut Gustave-Roussy, 53 Rue Camille Desmoulins, F-94800 Villejuif, France.

\section{References}

1 Mach JP, Carrel S, Merenda C, Sordat D, Cerottini JC. In-vivo localization of radiolabelled antibodies to carcinoembryonic antigen in human colon carcinoma grafted into nude mice. Nature 1974;248:704-6.

2 Goldenberg DM, Preston DF, Primus FJ, Hansen HJ. Photoscan localization of GW-39 tumors in hamsters using radiolabeled anticarcinoembryonic antigen immunoglobulin G. Cancer Res 1974;34:1-9.

${ }^{3}$ Hoffer PB, Lathrop K, Bekerman C, Fang VS, Refetoff S. Use of ${ }^{131}$ I-CEA antibody as a tumor scanning agent. $\mathcal{F}$ Nucl Med 1974;15:323-7.

${ }^{4}$ Goldenberg DM, Deland F, Kim E, et al. Use of radiolabeled antibody to carcino-embryonic antigen for detection and localization of diverse cancers by external photoscanning. $N$ Engl f Med 1978;298:1384-8.

${ }^{5}$ Mach JP, Carrel S, Forni M, Ritschard J, Donath A, Alberto P. Tumor localization of radiolabeled antibodies against carcinoembryonic antigen in patients with carcinoma, a critical evaluation. $N$ Engl 7 Med 1980; 303:5-10.
${ }^{6}$ Dykes PW, Hine KR, Bradwell AR, et al. Localization of tumour deposits by external scanning after injection of radiolabelled anti-carcinoembryonic antigen. Br.Med $\mathcal{F} 1980 ; \mathbf{2 8 0}: 220-2$.

${ }^{7}$ Goldenberg DM, Kim EE, Deland FH, Bennett S, Primus FJ. Radioimmunodetection of cancer with radioactive antibodies to carcinoembryonic antigen. Cancer Res 1980;40:2984-92.

${ }^{8}$ Mach JP, Forni M, Ritschard J, et al. Use and limitations of radiolabeled anti-CEA antibodies and their fragments for photoscanning detection in human colorectal carcinomas. Oncodevelopmental Biology and Medecine 1980;1:49-69.

${ }^{9}$ Accola RS, Carrel S, Mach JP. Monoclonal antibodies specific for carcinoembryonic antigen and produced by two hybrid cell lines. Proc Natl Acad Sci USA 1980;77:563-6.

${ }^{10}$ Mach JP, Buchegger F, Forni M, et al. Use of radiolabelled monoclonal anti-CEA antibodies for the detection of human carcinomas by external photoscanning and tomoscintigraphy. Immunology Today 1981 ;2:239-49.

11 Deland FH, Kim EE, Simmons G, Goldenberg DM. Imaging approach in radioimmunodetection. Cancer Res 1980;40:3046-9.

$12 \mathrm{Kuhl}$ DE, Edwards RD. Image separation radioisotope scanning. Radiology 1963;80:653-62.

13 Berche C, Aubert B, Bethencourt A, Di Paola R. Three-dimensional reconstruction based on sequential information from a scintillation camera. In: Brill AB, Price RR, eds. Information processing in medical imaging. Proceedings of the Vth International Conference. Nashville: Vanderbilt University, BCTIC, 1978:214-51.

14 Berche C, Aubry F, Langlais C, Vitaux J, Parmentier C, Di Paola R. Diagnostic value of transverse axial tomoscintigraphy for the detection of hepatic metastases: results on 53 examinations and comparison with other diagnostic techniques. Eur $\mathcal{F}$ Nucl Med 1981;6:435-52.

(Accepted 23 fuly 1982)

\title{
Association of specific immune response to pork and beef insulin with certain HLA-DR antigens in type 1 diabetes
}

\author{
I SKLENAR，T M NERI，W BERGER，P ERB
}

\begin{abstract}
To test the association of HLA-DR antigens with highresponder and low-responder status to either beef or pork insulin, insulin antibodies in diabetic sera were separated into those with average low and those with average high affinity and their insulin-binding capacities for each insulin determined. Significantly less binding of pork insulin by the high affinity antibodies occurred in the group of patients with DR3 antigens compared with those with DR4 antigens $(p<0.01)$ and $D R 3 / 4$ antigens $(p<0.01)$. The difference in the binding capacity of beef insulin by the high affinity antibodies between the groups with DR3 and DR4 antigens was less pronounced but still significant.
\end{abstract}

The high-responder status of DR3/4 antigens to pork insulin suggests that the gene or genes associated with HLA-DR4, and responsible for a high response to pork

\footnotetext{
Institute for Microbiology and Hygiene, University of Basel, Petersplatz 10, 4003 Basel, Switzerland

I SKLENAR, MD, research fellow

P ERB, PHD, chief of immunobiology

Basel Institute for Immunology, Switzerland

$T$ M NERI, MD, member of the institute

Department of Internal Medicine, University Hospital (Kantonsspital), Basel, Switzerland

W BERGER, MD, consultant physician
}

insulin, are dominant to genes associated with HLA-DR3 and a low response. If extended to human insulin and different HLA-DR and HLA-B antigen patterns, these findings should help in the therapeutic selection of the appropriate insulin and thus reduce the induction of an anti-insulin response in patients with diabetes.

\section{Introduction}

The immune response to insulin is under the control of genes, the so-called immune response genes, which are located within the major histocompatibility complex. Immune response genes to insulin have been most convincingly demonstrated in mice and guinea pigs, whereas their investigation in humans is far less advanced. Schernthaner et $a l^{1}$ and Bertrams et $a l^{2}$ correlated the high responder status of antibody production to insulin with the HLA-DR4 and B15 antigens, while DR3 and B8 antigens were more closely associated with a low responder status. Their particular technical approach did not allow discrimination between a high responder and low responder status with respect to the most commonly used insulins, beef and pork.

The insulin molecule is small and carries on its surface only a limited number of antigenic determinants. Furthermore, beef insulin differs from human insulin by three amino-acid exchanges: Ala for $\mathrm{Thr}$ at A8 (A-chain loop region), Val $\rightarrow \mathrm{Ile}$ at A10, and Ala $\rightarrow$ Thr at B30 (B-chain). Pork insulin differs only by one exchange: Ala $\rightarrow$ Thr at B30. To detect an antibody with binding specificity for one of these sites it is important that the antigen in the assay retains its native structure as far as possible. If the antibody-bound and free insulin is, however, 